\title{
Moderate quality evidence finds statistical benefit in oral health for powered over manual toothbrushes
}

\author{
Abstracted from \\ Yaacob M, Worthington HV, Deacon SA, Deery C, Walmsley AD, Robinson PG, Glenny AM. \\ Powered versus manual toothbrushing for oral health. Cochrane Database Syst Rev 2014; 6: Art. No. CD002281. \\ DOI: 10.1002/14651858.CD002281.pub3. \\ Address for correspondence: Cochrane Oral Health Group, School of Dentistry, \\ The University of Manchester, Coupland 3 Building, Oxford Road, Manchester, UK. M13 9PL \\ E-mail: cohg@manchester.ac.uk
}

\section{Question: Are powered toothbrushes as effective as manual toothbrushes in maintaining oral health?}

Data sources The Cochrane Oral Health Group's Trials Register, the Cochrane Central Register of Controlled Trials (CENTRAL), Medline, Embase, CINAHL, National Institutes of Health Trials Register and the WHO Clinical Trials Registry Platform for ongoing trials. Reference lists of identified articles were also scanned for relevant papers. Identified manufacturers were contacted for additional information.

Study selection Only randomised controlled trials comparing manual and powered toothbrushes were considered. Crossover trials were eligible for inclusion if the wash-out period length was more than two weeks.

Data extraction and synthesis Study assessment and data extraction were carried out independently by at least two reviewers. The primary outcome measures were quantified levels of plaque or gingivitis. Risk of bias assessment was undertaken. Standard Cochrane methodological approaches were taken. Random-effects models were used provided there were four or more studies included in the metaanalysis, otherwise fixed-effect models were used. Data were classed as short term (one to three months) and long term (greater than three months).

Results Fifty-six trials were included with 51 (4624 patients) providing data for meta-analysis. The majority (46) were at unclear risk of bias, five at high risk of bias and five at low risk. There was moderate quality evidence that powered toothbrushes provide a statistically significant benefit compared with manual toothbrushes with regard to the reduction of plaque in both the short and longterm. This corresponds to an $11 \%$ reduction in plaque for the Quigley Hein index (Turesky) in the short term and a $21 \%$ reduction in the long term. There was a high degree of heterogeneity that was not explained by the different powered toothbrush type subgroups.

There was also moderate quality evidence that powered toothbrushes again provide a statistically significant reduction in gingivitis when compared with manual toothbrushes both in the short and long term. This corresponds to a $6 \%$ and $11 \%$ reduction

This paper is based on a Cochrane Review published in the Cochrane Library 2014, issue 6 (see www.thecochranelibrary.com for information). Cochrane Reviews are regularly updated as new evidence emerges and in response to feedback, and the Cochrane Library should be consulted for the most recent version of the review. in gingivitis for the Löe and Silness indices respectively. Again there was a high degree of heterogeneity that was not explained by the different powered toothbrush type subgroups. The greatest body of evidence was for rotation oscillation brushes which demonstrated a statistically significant reduction in plaque and gingivitis at both time points.

Conclusions Powered toothbrushes reduce plaque and gingivitis more than manual toothbrushing in the short and long term. The clinical importance of these findings remains unclear. Observation of methodological guidelines and greater standardisation of design would benefit both future trials and meta-analyses. Cost, reliability and side effects were inconsistently reported. Any reported side effects were localised and only temporary.

\section{Commentary}

This systematic review assessed randomised controlled trials of the following types of power toothbrushes (compared to manual toothbrushes): side-to-side, counter-oscillation, rotation-oscillation, circular, ionic and ultrasonic.

The authors clearly state at the outset of their discussion that:

- 'The selection of one's toothbrush is largely a matter of personal preference, affordability, availability and professional recommendation.'

- There is overwhelming evidence that toothbrushing reduces gingivitis.' ${ }^{1}$

- 'These benefits occur whether the brush is manual or powered...'

All that said, the results of the very well done systematic review indicate that power toothbrushes, when compared to manual toothbrushes,

A. Reduce plaque by:

- $11 \%$ if used for less than three months (40 trials)

- $21 \%$ if used for greater than three months (14 trials)

B. Reduce gingivitis by:

- $6 \%$ if used for less than three months (44 trials)

- $11 \%$ if used for greater than three months (16 trials)

Of particular interest, and also consternation, is that only one powered toothbrush - rotating oscillating - consistently demonstrated significant improvement. All other power toothbrushes showed some statistical differences at some time points, but inconsistent differences at other time points.

One might expect manufacturers of power toothbrushes to create 
pristine clinical trials that support the superiority of their product. However, the quality of the trials was variable. Five trials were at high risk of bias, five at low risk of bias, and for the remaining 46 trials the risk of bias was unclear. Further, many trials used a split mouth design, which undermines generalisability, and many trials did not provide the following, which again raises concerns about validity:

- Power calculations

- Intention-to-treat analysis

- Evidence of adherence to CONSORT statement (http:// www.consort-statement.org/) guidelines, or adherence to recommendations for toothbrush trials. ${ }^{2}$

In spite of these concerns, the systematic review's authors found no evidence of publication bias. Further, they found no difference in outcomes based on sensitivity analysis of funding source for those trials with low risk of bias.

The sense one comes to is that power toothbrushes, as a class of toothbrush, offer a statistically significant reduction in plaque and gingivitis. And, when used for more than three months, this reduction may also be clinically significant.

Clinical significance is best understood from both the clinician's and patient's perspective. If plaque and gingivitis are not a significant clinical problem for a patient, then spending twice to ten-times the cost of a manual toothbrush to purchase a power toothbrush may be a step too far. On the other hand, if a patient has just invested $\$ 5,000, \$ 10,000$ or $\$ 20,000$ for restorative or aesthetic dentistry, then a $\$ 25$ to $\$ 100$ investment in a power toothbrush may be inexpensive insurance against plaque and gingivitis.

Finally, it is worth considering potential changes in methodologies and outcomes, given the 11-year time lapse from the first Cochrane review of power toothbrushes, ${ }^{3}$ and the current review. Over this period the number of subjects engaged in these toothbrush trials nearly doubled from approximately 2,500 to 4,500 , and the types of power toothbrushes increased by one (ionic).

However, the critiques of the individual trials remain essentially the same, as does the outcome - power toothbrushes appear to be more effective than manual - with the rotation-oscillation appearing to be more consistently effective than the other power toothbrushes. ${ }^{4}$ This suggests that the Cochrane Oral Health Group may want to consider using methods for assessing need for a new review ${ }^{5,6}$ as an alternate to the stated two-year time frame. ${ }^{7}$ This would simultaneously conserve resources and provide miniupdates. An oral health example of this was recently provided with good effect in examining the need for further systematic reviews comparing glass-ionomer and resin sealants. ${ }^{8}$

Richard Niederman

Department of Epidemiology and Health Promotion, Director, Center for Evidence-Based Dentistry, College of Dentistry, New York University, New York, USA

1. Lang NP, Cumming BR, Löe $H$. Toothbrushing frequency as it relates to plaque development and gingival health. J Periodontol 1973; 44: 396-405.

2. Robinson PG, Damien Walmsley A, Heanue M, et al. Quality of trials in a systematic review of powered toothbrushes: suggestions for future clinical trials. J Periodontol 2006; 77: 1944-1953.

3. Deery $C$, Heanue $M$, Deacon $S$, et al. The effectiveness of manual versus powered toothbrushes for dental health: a systematic review. I Dent 2004; 32: 197-211.

4. Niederman R; ADA Council on Scientific Affairs; ADA Division of Science; Journal of the American Dental Association. Manual versus powered toothbrushes: the Cochrane review. J Am Dent Assoc 2003; 134: 1240-1244.

5. Chung M, Newberry SJ, Ansari MT, et al. Two methods provide similar signals for the need to update systematic reviews. / Clin Epidemiol 2012; 65: 660-668.

6. Shojania KG, Sampson M, Ansari MT, Ji J, Doucette S, Moher D. How quickly do systematic reviews go out of date? A survival analysis. Ann Intern Med 2007; 147: 224-233.

7. Higgins JPT, Green S (editors). Cochrane Handbook for Systematic Reviews of Interventions, Section 3.4.1. Version 5.1.0 [updated March 2011]. The Cochrane Collaboration, 2011. Available from www.cochrane-handbook.org.

8. Mickenautsch S, Yengopal V. The modified Ottawa method to establish the update need of a systematic review: glass-ionomer versus resin sealants for caries prevention. J Appl Oral Sci 2013; 21: 482-489.

Evidence-Based Dentistry (2014) 15, 77-78. doi:10.1038/sj.ebd.6401041 